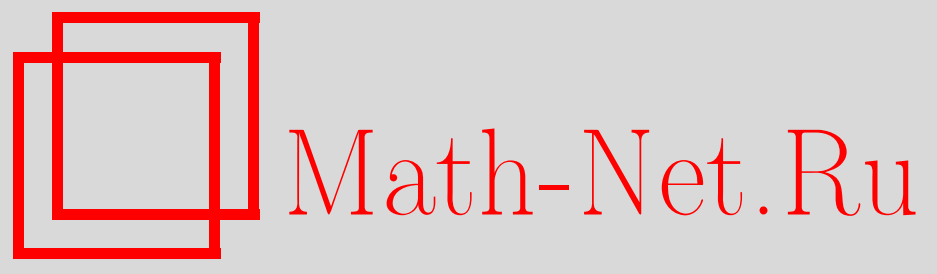

В. А. Кудинов, А. Э. Кузнецова, А. В. Еремин, Е. В. Котова, Аналитические решения задач термоупругости для многослойных конструкций с переменными свойствами, Вестн. Сам. гос. техн. ун-та. Сер. Физ.-мат. науки, 2013, выпуск 1(), 215-221

DOI: https://doi.org/10.14498/vsgtu1128

Использование Общероссийского математического портала Math-Net.Ru подразумевает, что вы прочитали и согласны с пользовательским соглашением

http: //www . mathnet.ru/rus/agreement

Параметры загрузки:

IP : 54.224 .135 .184

26 апреля 2023 г., 15:26:18

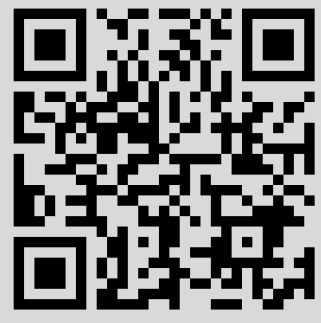


Вестн. Сам. гос. техн. ун-та. Сер. Физ.-мат. науки. 2013. № 1 (30). С. $215-221$

УДК 517.958:539.3(4)

\title{
АНАЛИТИЧЕСКИЕ РЕШЕНИЯ ЗАДАЧ ТЕРМОУПРУГОСТИ ДЛЯ МНОГОСЛОЙНЫХ КОНСТРУКЦИЙ С ПЕРЕМЕННЫМИ СВОЙСТВАМИ
}

\author{
В.А. Кудинов, А.Э. Кузнецова, А.В. Ерёмин, Е. В. Котова \\ Самарский государственный технический университет, \\ Россия, 443100, Самара, ул. Молодогвардейская, 244. \\ E-mails: kud-samgtu@yandex.ru, a.v.eremin@list.ru \\ Разработана методика получения приближённых аналитических решений ква- \\ зистатических задач термоупругости (плоское напряжённое состояние, плос- \\ кая деформачия) для многослойных конструкиий с переменными в пределах каж- \\ дого слоя физическими свойствами среды. Использован рекуррентный метод по- \\ строения систем координатных функиий, точно удовлетворяющих граничным \\ условиям сопряжения, заданным в виде равенства радиальных (нормальных) на- \\ пряжений и перемещений в точках контакта слоёв.
}

Ключевые слова: многослойные конструкции, аналитическое решение, задача термоупругости, переменные физические свойства среды, система координатных функиий, ортогональный метод Бубнова-Галёркина.

Точные аналитические решения задач термоупругости с переменными в пределах каждого слоя физическими свойствами среды (применительно к задачам в квазистатической постановке, то есть без учёта изменения напряжений во времени) в настоящее время не получены. Основная причина заключается в нелинейности исходной системы обыкновенных дифференциальных уравнений, а также в необходимости выполнения не только граничных условий, но и условий сопряжения между слоями, заданных в виде равенства напряжений и перемещений в точках контакта слоёв.

В настоящей работе применительно к решению указанных задач разработан рекуррентный метод построения систем координатных функций, точно удовлетворяющих граничным условиям и условиям сопряжения. Его основная идея состоит в последовательном построении координатных функций для каждого тела при использовании метода неопределенных коэффициентов.

Выполнение системы исходных дифференциальных уравнений осуществлялось путём составления их невязки и требования ортогональности невязки ко всем координатным функциям (ортогональный метод Бубнова-Галёркина). При этом решение системы обыкновенных дифференциальных уравнений сводится к решению системы алгебраических линейных уравнений относительно неизвестных коэффициентов, число которых равно числу приближений. Реализация такого метода решения оказывается возможной благодаря использованию глобальной (одинаковой для всех слоев) системы неизвестных коэффициентов, содержащихся в решениях для каждого отдельного слоя.

Василий Александрович Кудинов (д.ф.-м.н., проф.), заведующий кафедрой, каф. теоретических основ теплотехники и гидромеханики.

Анастасия Эдуардовна Кузнецова, ассистент, каф. теоретических основ теплотехники и гидромеханики. Антон Владимирович Ерёмин, ассистент, каф. теоретических основ теплотехники и гидромеханики. Евгения Валериевна Котова, ассистент, каф. теоретических основ теплотехники и гидромеханики. 
Система дифференциальных уравнений относительно радиального перемещения для случая, когда модуль упругости и коэффициент линейного расширения в пределах каждого слоя являются произвольными функциями радиуса (рассматривается длинный полый цилиндр - плоская деформация), имеет вид $[1,2]$

$$
\begin{aligned}
\frac{d}{d r}\left(E_{i} r \frac{d U_{i}}{d r}\right)+ & \left(\frac{\nu_{i}}{1-\nu_{i}} \frac{d E_{i}}{d r}-\frac{E_{i}}{r}\right) U_{i}=\frac{\left(1+\nu_{i}\right) r}{1-v_{i}} \frac{d}{d r}\left(\alpha_{i} E_{i} T_{i}\right), \\
& r_{i}<r<r_{i+1}, \quad i=1,2, \ldots, m,
\end{aligned}
$$

где $U_{i}(r)$ - радиальное перемещение $i$-того слоя; $E_{i}(r), \alpha_{i}(r), T_{i}(r)$ - соответствующие $i$-тому слою модуль упругости, коэффициент линейного расширения и температура; $\nu_{i}$ - коэффициент Пуассона $i$-того слоя; $r$ - радиальная координата; $m$ - число слоёв.

Для случая, когда $E_{i}=$ const и $\alpha_{i}=$ const, система уравнений (1) приводится к виду

$$
\frac{d^{2} U_{i}}{d r^{2}}+\frac{1}{r} \frac{d U_{i}}{d r}-\frac{U_{i}}{r^{2}}-\frac{1+\nu_{i}}{1-\nu_{i}} \alpha_{i} \frac{d T_{i}}{d r}=0, \quad r_{i}<r<r_{i+1}, \quad i=1,2, \ldots, m .
$$

Напряжения в каждом слое по известному перемещению определяются по формулам

$$
\begin{aligned}
& \sigma_{r i}=\frac{E_{i}}{\left(1+\nu_{i}\right)\left(1-2 \nu_{i}\right)}\left[\left(1-\nu_{i}\right) \frac{d U_{i}}{d r}+\nu_{i} \frac{U_{i}}{r}-\left(1+\nu_{i}\right) \alpha_{i} T_{i}\right] \\
& \sigma_{\theta i}=\frac{E_{i}}{\left(1+\nu_{i}\right)\left(1-2 \nu_{i}\right)}\left[\nu_{i} \frac{d U_{i}}{d r}+\left(1-\nu_{i}\right) \frac{U_{i}}{r}-\left(1+\nu_{i}\right) \alpha_{i} T_{i}\right],
\end{aligned}
$$

где $\sigma_{r i}, \sigma_{\theta i}$ - радиальное и окружное напряжения в $i$-том слое.

$\mathrm{C}$ целью упрощения математических преобразований основную идею метода рассмотрим на примере решения задачи термоупругости для двухслойного длинного полого цилиндра $(m=2)$ с постоянными в пределах каждого слоя физическими свойствами среды. При этом отметим, что без каких-либо изменений этот метод может быть применён и к решению задач термоупругости для многослойных конструкций с переменными свойствами.

Граничные условия применительно к двухслойному полому цилиндру имеЮт вид

$$
\begin{gathered}
{\left[a_{1} \frac{d U_{1}}{d r}+\nu_{1} \frac{U_{1}}{r}\right]_{r=r_{1}}=\left.c_{1} \alpha_{1} T_{1}\right|_{r=r_{1}},} \\
{\left[a_{2} \frac{d U_{2}}{d r}+\nu_{2} \frac{U_{2}}{r}\right]_{r=r_{3}}=\left.c_{2} \alpha_{2} T_{2}\right|_{r=r_{3}} ;} \\
\eta_{1} E_{1}\left[a_{1} \frac{d U_{1}}{d r}+\nu_{1} \frac{U_{1}}{r}-c_{1} \alpha_{1} T_{1}\right]_{r=r_{2}}=\eta_{2} E_{2}\left[a_{2} \frac{d U_{2}}{d r}+\nu_{2} \frac{U_{2}}{r}-c_{2} \alpha_{2} T_{2}\right]_{r=r_{2}} ; \\
U_{1}\left(r_{2}\right)=U_{2}\left(r_{2}\right),
\end{gathered}
$$

где $a_{1}=1-\nu_{1} ; a_{2}=1-\nu_{2} ; c_{1}=1+\nu_{1} ; c_{2}=1+\nu_{2} ; \eta_{1}=\left[\left(1+\nu_{1}\right)\left(1-2 \nu_{1}\right)\right]^{-1}$; $\eta_{2}=\left[\left(1+\nu_{2}\right)\left(1-2 \nu_{2}\right)\right]^{-1}$. 
Из соотношений (5), (6) следует, что радиальные напряжения на внутренней $\left(r=r_{1}\right)$ и внешней $\left(r=r_{3}\right)$ поверхностях цилиндра равны нулю. Соотношения (7), (8) представляют собой условия сопряжения между слоями, записанные в виде равенства радиальных напряжений (соотношение (7)) и перемещений (соотношение (8)) в точке контакта слоёв $r=r_{2}$.

С целью упрощения процесса получения решения приведем задачу (2)-(8) к безразмерному виду. Для этого введём следующие безразмерные переменные и параметры: $\vartheta_{i}=U_{i} / U_{\mathrm{M}}, \rho=r / r_{3}, \rho_{1}=r_{1} / r_{3}, \rho_{2}=r_{2} / r_{3}, \rho_{3}=r_{3} / r_{3}=1$, $\varepsilon_{1}=E_{1} / E_{1}=1, \varepsilon_{2}=E_{2} / E_{1}, \mu_{1}=\alpha_{1} / \alpha_{1}=1, \mu_{2}=\alpha_{2} / \alpha_{1} ; \Theta_{i}=T_{i} / T_{\mathrm{M}}, \sigma_{r i}^{*}=$ $=\sigma_{r i}^{*} / \sigma_{\mathrm{M}}, \sigma_{\theta i}^{*}=\sigma_{\theta i} / \sigma_{\mathrm{M}} ; i=1,2$, где $U_{\mathrm{M}}, T_{\mathrm{M}}, \sigma_{\mathrm{M}}$ - масштабные перемещение и температура.

С учётом принятых обозначений задача (2), (5)-(8) принимает вид

$$
\begin{gathered}
\frac{d^{2} \vartheta_{i}}{d \rho^{2}}+\frac{1}{\rho} \frac{d \vartheta_{i}}{d \rho}-\frac{\vartheta_{i}}{\rho^{2}}-\beta_{i} \mu_{i} \alpha_{1} r_{3} \frac{T_{\mathrm{M}}}{U_{\mathrm{M}}} \frac{d \Theta_{i}}{d \rho}=0 \\
{\left[a_{1} \frac{d \vartheta_{1}}{d \rho}+\nu_{1} \frac{\vartheta_{1}}{\rho}\right]_{\rho=\rho_{1}}=\left.\frac{c_{1} \mu_{1} \alpha_{1} r_{3} T_{\mathrm{M}}}{U_{\mathrm{M}}} \Theta_{1}\right|_{\rho=\rho_{1}}} \\
{\left[a_{2} \frac{d \vartheta_{2}}{d \rho}+\nu_{2} \frac{\vartheta_{2}}{\rho}\right]_{\rho=1}=\left.\frac{c_{2} \mu_{2} \alpha_{1} r_{3} T_{\mathrm{M}}}{U_{\mathrm{M}}} \Theta_{2}\right|_{\rho=1} ;} \\
\eta_{1} \varepsilon_{1}\left[a_{1} \frac{d \vartheta_{1}}{d \rho}+\nu_{1} \frac{\vartheta_{1}}{\rho}-\frac{c_{1} \mu_{1} \alpha_{1} r_{3} T_{\mathrm{M}}}{U_{\mathrm{M}}} \Theta_{1}\right]_{\rho=\rho_{2}}= \\
=\eta_{2} \varepsilon_{2}\left[a_{2} \frac{d \vartheta_{2}}{d \rho}+\nu_{2} \frac{\vartheta_{2}}{\rho}-\frac{c_{2} \mu_{2} \alpha_{1} r_{3} T_{\mathrm{M}}}{U_{\mathrm{M}}} \Theta_{2}\right]_{\rho=\rho_{2}} ; \\
\vartheta_{1}\left(\rho_{2}\right)=\vartheta_{2}\left(\rho_{2}\right),
\end{gathered}
$$

где $\beta_{i}=\left(1+\nu_{i}\right) /\left(1-\nu_{i}\right) ; i=1,2$.

Формулы (3), (4) в безразмерном случае примут вид

$$
\begin{aligned}
\sigma_{r i}^{*} & =\frac{\eta_{i} \varepsilon_{i}}{\sigma_{\mathrm{M}}}\left[a_{i} \frac{d \vartheta_{i}(\rho)}{d \rho}+\nu_{i} \frac{\vartheta_{i}(\rho)}{\rho}-\frac{c_{i} \mu_{i} \alpha_{1} r_{3} T_{\mathrm{M}}}{U_{\mathrm{M}}} \Theta_{i}(\rho)\right] \\
\sigma_{\theta i}^{*} & =\frac{\eta_{i} \varepsilon_{i}}{\sigma_{\mathrm{M}}}\left[\nu_{i} \frac{d \vartheta_{i}(\rho)}{d \rho}+a_{i} \frac{\vartheta_{i}(\rho)}{\rho}-\frac{c_{i} \mu_{i} \alpha_{1} r_{3} T_{\mathrm{M}}}{U_{\mathrm{M}}} \Theta_{i}(\rho)\right] .
\end{aligned}
$$

В качестве модельного примера предположим, что температура в каждом слое описывается функцией

$$
T_{i}(r)=D_{i}+R_{i} r \quad(i=1,2),
$$

где $D_{1}=140,32 ; R_{1}=-2880 ; D_{2}=53,375 ; R_{2}=-625$.

Соотношение (14) в безразмерном виде для каждого слоя запишется так:

$$
\theta_{1}(\rho)=1,4032-1,584 \rho, \quad \theta_{2}(\rho)=0,53375-0,34375 \rho .
$$

Решение задачи (9)-(13) принимается в виде [3]

$$
\vartheta_{1}(\rho)=\Phi_{1}(\rho)+\sum_{k=2}^{n} q_{k} \varphi_{1 k}(\rho),
$$




$$
\vartheta_{2}(\rho)=\Phi_{2}(\rho)+\sum_{k=2}^{n} q_{k} \varphi_{2 k}(\rho)
$$

где $\Phi_{1}(\rho)=F_{1}+F_{2} \rho+F_{3} \rho^{2}, \Phi_{2}(\rho)=F_{4} \rho-$ функции, неизвестные коэффициенты $F_{i}$ которых определяются так, чтобы выполнялись неоднородные граничные условия (10), (11) и условия сопряжения (12), (13); $\varphi_{1 k}=B_{1 k}+$ $+B_{2 k} \rho+B_{3 k} \rho^{k}, \varphi_{2 k}=1+B_{4 k} \rho^{k}$ - координатные функции, неизвестные коэффициенты $B_{i k}$ которых находятся из однородных граничных условий (10) и условий сопряжения (12), (13), то есть при равенстве нулю всех членов, находящихся в произведении с $\theta_{1}(\rho)$ и $\theta_{2}(\rho) ; q_{k}$ - неизвестные коэффициенты (одинаковые для каждого слоя), определяемые из выполнения дифференциальных уравнений (9).

Рассмотрим последовательность определения функций $\Phi_{1}(\rho)$ и $\Phi_{2}(\rho)$. Сначала находится функция $\Phi_{2}(\rho)$, неизвестный коэффициент $F_{4}$ которой определяется так, чтобы выполнялось неоднородное граничное условие (11). Подставляя $\Phi_{2}(\rho)$ в $(11)$, находим

$$
F_{4}=\frac{c_{2} \mu_{2} \alpha_{1} r_{3} T_{\mathrm{M}}}{U_{\mathrm{M}}\left(a_{2}+\nu_{2}\right)} \Theta_{2}(1) .
$$

Неизвестные коэффициенты $F_{1}, F_{2}, F_{3}$ функции $\Phi_{1}(\rho)$ находятся так, чтобы выполнялось неоднородное граничное условие (10) и условия сопряжения $(12),(13)$. Подставляя $\Phi_{1}(\rho)$ и $\Phi_{2}(\rho)$ в $(10),(12),(13)$, относительно коэффициентов $F_{1}, F_{2}, F_{3}$ получаем следующую систему трёх алгебраических линейных уравнений:

$$
\begin{aligned}
& {\left[a_{1} \frac{d \Phi_{1}(\rho)}{d \rho}+\nu_{1} \frac{\Phi_{1}(\rho)}{\rho}-\frac{c_{1} \mu_{1} \alpha_{1} r_{3} T_{\mathrm{M}}}{U_{\mathrm{M}}} \Theta_{1}(\rho)\right]_{\rho=\rho_{1}}=0 ;} \\
& \eta_{1} \varepsilon_{1}\left[a_{1} \frac{d \Phi_{1}(\rho)}{d \rho}+\nu_{1} \frac{\Phi_{1}(\rho)}{\rho}-\frac{c_{1} \mu_{1} \alpha_{1} r_{3} T_{\mathrm{M}}}{U_{\mathrm{M}}} \Theta_{1}(\rho)\right]_{\rho=\rho_{2}}= \\
& =\eta_{2} \varepsilon_{2}\left[a_{2} \frac{d \Phi_{2}(\rho)}{d \rho}+\nu_{2} \frac{\Phi_{2}(\rho)}{\rho}-\frac{c_{2} \mu_{2} \alpha_{1} r_{3} T_{\mathrm{M}}}{U_{\mathrm{M}}} \Theta_{2}(\rho)\right]_{\rho=\rho_{2}} ; \\
& \Phi_{1}\left(\rho_{2}\right)-\Phi_{2}\left(\rho_{2}\right)=0 .
\end{aligned}
$$

Неизвестный коэффициент $B_{4 k}$ координатных функций $\varphi_{2 k}(\rho)$ находится из однородного граничного условия (11). Подставляя $\varphi_{2 k}(\rho)$ в $(11)$, положив правую часть равной нулю, получаем

$$
\left[a_{2} \frac{d \varphi_{2 k}(\rho)}{d \rho}+\nu_{2} \frac{\varphi_{2 k}(\rho)}{\rho}\right]_{\rho=1}=0, \quad k=2,3, \ldots, n .
$$

Из решения алгебраического уравнения (17) находим

$$
B_{4 k}=-\nu_{2} /\left(\nu_{2}+k a_{2}\right) .
$$

Неизвестные коэффициенты $B_{1 k}, B_{2 k}, B_{3 k}$ координатных функций $\varphi_{1 k}(\rho)$ находятся из однородного граничного условия (10) и однородных условий сопряжения $(12),(13)$. Подставляя $\varphi_{1 k}(\rho)$ и $\varphi_{2 k}(\rho)$ в $(10),(12),(13)$, положив 
члены, находящиеся в произведении с $\Theta_{1}(\rho)$ и $\Theta_{2}(\rho)$, равными нулю, относительно неизвестных коэффициентов $B_{1 k}, B_{2 k}, B_{3 k}$ получаем следующую систему трёх алгебраических линейных уравнений:

$$
\begin{aligned}
& {\left[a_{1} \frac{d \varphi_{1 k}(\rho)}{d \rho}+\nu_{1} \frac{\varphi_{1 k}(\rho)}{\rho}-\frac{c_{1} \mu_{1} \alpha_{1} r_{3} T_{\mathrm{M}}}{U_{\mathrm{M}}} \Theta_{1}(\rho)\right]_{\rho=\rho_{1}}=0} \\
& \eta_{1} \varepsilon_{1}\left[a_{1} \frac{d \varphi_{1 k}(\rho)}{d \rho}+\nu_{1} \frac{\varphi_{1 k}(\rho)}{\rho}-\frac{c_{1} \mu_{1} \alpha_{1} r_{3} T_{\mathrm{M}}}{U_{\mathrm{M}}} \Theta_{1}(\rho)\right]_{\rho=\rho_{2}}= \\
& =\eta_{2} \varepsilon_{2}\left[a_{2} \frac{d \varphi_{2 k}(\rho)}{d \rho}+\nu_{2} \frac{\varphi_{2 k}(\rho)}{\rho}-\frac{c_{2} \mu_{2} \alpha_{1} r_{3} T_{\mathrm{M}}}{U_{\mathrm{M}}} \Theta_{2}(\rho)\right]_{\rho=\rho_{2}} \\
& \varphi_{1 k}\left(\rho_{2}\right)-\varphi_{2 k}\left(\rho_{2}\right)=0 .
\end{aligned}
$$

После нахождения коэффициентов $F_{i}$ и $B_{i k}$ соотношения $(15),(16)$ при любых значениях неизвестных коэффициентов $q_{k}$ будут точно удовлетворять граничным условиям (10), (11) и условиям сопряжения (12), (13). Для определения неизвестных коэффициентов $q_{k}$ составим невязки уравнений (9) и потребуем ортогональности невязок ко всем координатным функциям $\varphi_{1 k}(\rho)$ и $\varphi_{2 k}(\rho)$ :

$$
\begin{aligned}
\int_{\rho_{1}}^{\rho_{2}}\left(\frac{d^{2} \vartheta_{1}}{d \rho^{2}}\right. & \left.+\frac{1}{\rho} \frac{d \vartheta_{1}}{d \rho}-\frac{\vartheta_{1}}{\rho^{2}}-\beta_{1} \mu_{1} \alpha_{1} r_{3} \frac{T_{\mathrm{M}}}{U_{\mathrm{M}}} \frac{d \Theta_{1}}{d \rho}\right) \varphi_{1 j}(\rho) \rho d \rho+ \\
& +\int_{\rho_{2}}^{1}\left(\frac{d^{2} \vartheta_{2}}{d \rho^{2}}+\frac{1}{\rho} \frac{d \vartheta_{2}}{d \rho}-\frac{\vartheta_{2}}{\rho^{2}}-\beta_{2} \mu_{2} \alpha_{1} r_{3} \frac{T_{\mathrm{M}}}{U_{\mathrm{M}}} \frac{d \Theta_{2}}{d \rho}\right) \varphi_{2 j}(\rho) \rho d \rho=0
\end{aligned}
$$

$j=2,3, \ldots, n$.

Подставляя (15), (16) в (18), после определения интегралов относительно неизвестных коэффициентов $q_{k}$ будем иметь систему $n-2$ алгебраических линейных уравнений.

Исходные данные для решения задачи следующие [3-5]: $r_{1}=0,014 \mathrm{~m} ; r_{2}=$ $=0,039 \mathrm{~m} ; r_{3}=0,055 \mathrm{~m} ; v_{1}=v_{2}=0,2 ; E_{1}=19,5 \cdot 10^{9} \mathrm{K \Gamma} / \mathrm{m}^{2} ; \sigma_{\mathrm{M}}=0,1107 ;$ $E_{2}=13 \cdot 10^{9} \mathrm{Kг} / \mathrm{M}^{2} ; \alpha_{1}=15 \cdot 10^{-6} / \mathrm{K} ; \alpha_{2}=11 \cdot 10^{-6} / \mathrm{K} ; T_{\mathrm{M}}=100^{\circ} \mathrm{C} ; U_{\mathrm{M}}=10^{-3} \mathrm{M}$.

Результаты расчётов безразмерных радиальных и окружных напряжений по формулам (15), (16) во втором приближении даны на рис. 1. Отметим, что соотношения (15), (16) в данном случае точно удовлетворяют граничным

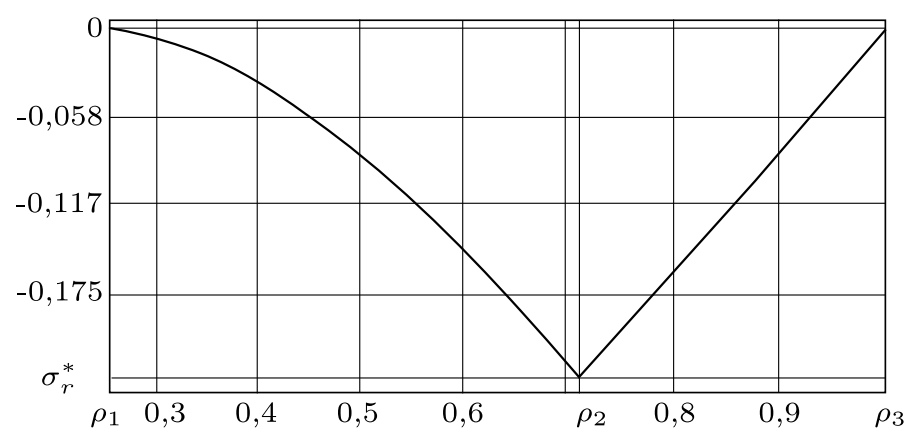

Рис. 1. Изменение радиальных напряжений (второе приближение) 
условиям и условиям сопряжения (10)-(13), точность выполнения уравнений (9) зависит от числа приближений (от числа членов рядов (15), (16)).

Результаты расчётов напряжений $\sigma_{r i}^{*}$ и $\sigma_{\theta i}^{*}$ даны на рис. 1,2 . Их анализ позволяет сделать вывод о том, что радиальные напряжения $\sigma_{r}^{*}$ имеют отрицательный знак (сжатие). Радиальные напряжения на внешних поверхностях стенок $\left(\rho=\rho_{1}\right.$ и $\left.\rho=1\right)$, как это следует из граничных условий $(10)$, (11), равны нулю. Окружные напряжения $\sigma_{\theta}^{*}$ в пределах первого слоя имеют отрицательный знак, а в пределах второго - положительный (растяжение). В точке контакта слоёв $\left(\rho=\rho_{2}\right)$ ввиду различия числовых значений модулей упругости и коэффициентов линейного расширения, а также вследствие непрерывности перемещений наблюдается скачок первого рода для функции окружных напряжений.

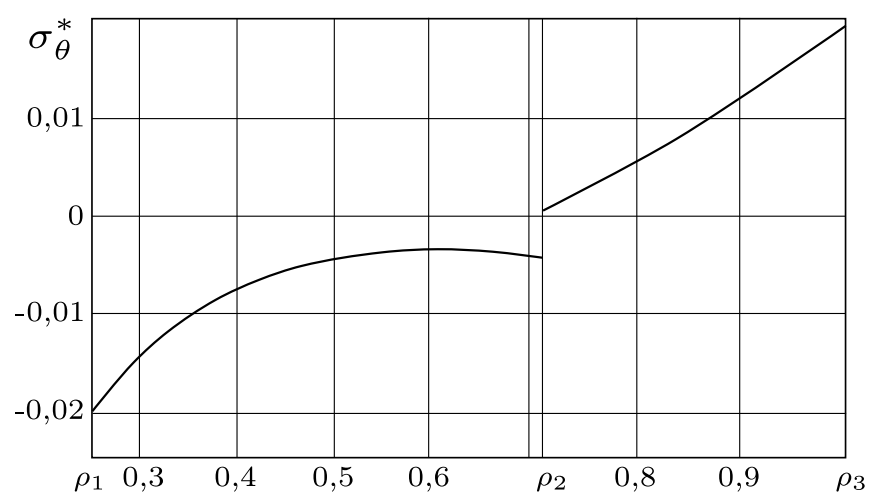

Рис. 2. Изменение окружных напряжений (второе приближение)

Заключение. На основе использования систем координатных функций, точно удовлетворяющих граничным условиям и условиям сопряжения, разработана методика получения приближённых аналитических решений квазистатических задач термоупругости для многослойных конструкций на примере цилиндра с переменными в пределах каждого слоя физическими свойствами среды. Отличительной особенностью метода является использование глобальной (одинаковой для каждого слоя) системы неизвестных коэффициентов, позволяющей с помощью ортогонального метода Бубнова-Галёркина свести решение исходной системы обыкновенных дифференциальных уравнений к решению системы алгебраических линейных уравнений, число неизвестных которой (принятых коэффициентов $q_{k}$ ) равно числу приближений.

\section{БИБЛИОГРАФИЧЕСКИЙ СПИСОК}

1. А. Д. Коваленко, Введение в термоупругость. Киев: Наук. думка, 1965. 202 с. [A. D. Kovalenko, Introduction to Thermoelasticity. Kiev: Nauk. Dumka, 1965. 202 pp.]

2. S. Timoshenko, J. N. Goodier, Theory of Elasticity. New York, Toronto, London: McGrawHill Book Company, Inc., 1951. xviii+506 pp.; русск. пер.: С. П. Тимошенко, Дж. Гудъер, Теория упругости. М.: Наука, 1979. 560 с.

3. Кудинов В. А., Карташов Э. М., Калашников В. В., Аналитические решения задач тепломассопереноса и термоупругости для многослойных конструкций. М.: Высш. шк., 2005. 430 c. [V. A. Kudinov, É. M. Kartashov, V. V. Kalashnikov, Analytical solutions of problem of heat and mass transfer and thermoelasticity for multilayered structures. Moscow: Vyssh. shk., 2005. 430 pp.] 
4. В. А. Кудинов, А. В. Еремин, Е. В. Котова, "Получение аналитических решений задач термоупругости для многослойных тел с переменными свойствами" / В сб.: Третья международная конферениия «Математическая физика и её приложения»: Материалы конф. (Самара, 27 августа - 1 сентября, 2012 г.); ред. чл.-корр. РАН И. В. Волович, д.ф.-м.н., проф. В. П. Радченко. Самара: СамГТУ, 2012. С. 184-185. [V. A. Kudinov, A. V. Eremin, E. V. Kotova, "Analytical solutions of problems of thermoelasticity for multilayered bodies with variable properties" / In: The Third International Conference "Mathematical Physics and Its Applications": Book of Abstracts (August 27 - September 01, 2012 Samara, Russia); eds. I. V. Volovich, V. P. Radchenko. Samara: Samara State Technical Univ., 2012. Pp. 184-185].

5. В. А. Кудинов, А. В. Еремин, Е. В. Котова, "Получение точных аналитических решений задач термоупругости для многослойных конструкций" // Вестн. Сам. гос. техн. ун-та. Сер. Физ.-мат. науки, 2012. №2(27). C. 188-191. [V. A. Kudinov, A. V. Eremin, E. V. Kotova, "Obtaining exact analytical solutions of the thermoelasticity problem for multilayer cylindrical structures"// Vestn. Samar. Gos. Tekhn. Univ. Ser. Fiz.-Mat. Nauki, 2012. no. 2(27). Pp. 188-191].

Поступила в редакцию $29 / \mathrm{X} / 2012$;

в окончательном варианте - 01/II/2013.

MSC: 74F05; 74C05, 80A20

\title{
ANALYTICAL SOLUTIONS OF PROBLEMS OF THERMOELASTICITY FOR MULTILAYERED BODIES WITH VARIABLE PROPERTIES
}

\author{
V. A. Kudinov, A.E. Kuznetsova, A. V. Eremin, E. V. Kotova
}

Samara State Technical University,

244, Molodogvardeyskaya st., Samara, 443100, Russia.

E-mails: kud-samgtu@yandex.ru, a.v.eremin@list.ru

The technics for the construction of approximate analytical solutions for the quasistatic problems of thermoelasticity (plane-stressed state, plane deformation) for the multilayered bodies with variable within limits of each layer physical properties of medium. The recursive method is used for the construction of systems of coordinate functions, satisfying the boundary matching conditions, given as the equality of radial (normal) stresses and displacements in the layer-contact points.

Key words: multilayer constructions, analytical solution, thermoelasticity problem, environmental variable physical properties, system of coordinate functions, BubnovGalyorkin orthogonal method.

Original article submitted 29/X/2012; revision submitted 01/II/2013.

Vasiliy A. Kudinov (Dr. Sci. (Phys. \& Math.)), Head of Dept., Dept. of Theoretical Basis of Heat Engineering \& Flow Mechanics.

Anastasiya E. Kuznetsova, Assistent, Dept. of Theoretical Basis of Heat Engineering \& Flow Mechanics. Anton V. Eremin, Assistent, Dept. of Theoretical Basis of Heat Engineering \& Flow Mechanics. Evgeniya V. Kotova, Assistent, Dept. of Theoretical Basis of Heat Engineering \& Flow Mechanics. 